\title{
On Some Properties and Symmetries of the 5-Dimensional Lorenz System
}

\author{
Cristian Lăzureanu and Tudor Bînzar \\ Department of Mathematics, Politehnica University of Timişoara, Piaţa Victoriei No. 2, 300006 Timişoara, Romania \\ Correspondence should be addressed to Cristian Lăzureanu; cristian.lazureanu@upt.ro
}

Received 11 August 2015; Revised 3 October 2015; Accepted 5 October 2015

Academic Editor: Yan-Wu Wang

Copyright (C) 2015 C. Lăzureanu and T. Bînzar. This is an open access article distributed under the Creative Commons Attribution License, which permits unrestricted use, distribution, and reproduction in any medium, provided the original work is properly cited.

The 5-dimensional Lorenz system for the gravity-wave activity is considered. Some stability problems and the existence of periodic orbits are studied. Also, a symplectic realization and some symmetries are given.

\section{Introduction}

The importance of the 5-dimensional Lorenz system [1] in the study of geophysical fluid dynamics is well known. This system describes coupled Rossby waves and gravity waves. It was mainly investigated from the existence of a slow manifold point of view [2-5]. Among other studies regarding 5-dimensional Lorenz system we mention Hamiltonian structure [6], chaotic behaviour [7-9], and analytic integrability [10].

According to [10], the 5-dimensional Lorenz system has at most three functionally independent global analytic first integrals. We mention that two first integrals are known [1]. It raises the following question: how can the third first integral be determined, provided that it exists? A possible answer is given by the connection between symmetries and the existence of conservative laws [11]. Our main purpose is to try to determine the third first integral using symmetries. This attempt was successful in the case of 5-dimensional MaxwellBloch equations with the rotating wave approximation [12]. "Intuitively speaking, a symmetry is a transformation of an object leaving this object invariant" [13]. In our case, a transformation means a vector field and an object means a differential equation. Recently, this field is widely investigated. We refer to some new progress [14-17].

In our paper, the constants of motion of the 5-dimensional Lorenz system are used to study some stability problems and the existence of periodic orbits. "The stability of an orbit of a dynamical system characterizes whether nearby (i.e., perturbed) orbits will remain in a neighborhood of that orbit or be repelled away from it" [18]. Also, with the aid of these constants of motion, a symplectic realization and a Lagrangian formulation are given. In the last part of our work some symmetries are pointed out.

\section{Stability and Periodic Orbits}

We consider the 5-dimensional Lorenz system [1]:

$$
\begin{aligned}
& \dot{x}_{1}=-x_{2} x_{3}+b x_{2} x_{5}, \\
& \dot{x}_{2}=x_{1} x_{3}-b x_{1} x_{5}, \\
& \dot{x}_{3}=-x_{1} x_{2}, \\
& \dot{x}_{4}=-x_{5}, \\
& \dot{x}_{5}=x_{4}+b x_{1} x_{2},
\end{aligned}
$$

where $b \in \mathbb{R}$.

Recall that, for system (1), the functions $H, C \in$ $\mathscr{C}^{\infty}\left(\mathbb{R}^{5}, \mathbb{R}\right)$

$$
\begin{aligned}
& H\left(x_{1}, x_{2}, x_{3}, x_{4}, x_{5}\right)=\frac{1}{2}\left(x_{1}^{2}+2 x_{2}^{2}+x_{3}^{2}+x_{4}^{2}+x_{5}^{2}\right), \\
& C\left(x_{1}, x_{2}, x_{3}, x_{4}, x_{5}\right)=\frac{1}{2}\left(x_{1}^{2}+x_{2}^{2}\right)
\end{aligned}
$$


are constants of motion. The functions $H$ and $C$ are linearly related to analogs of the energy and, respectively, enstrophy of the nine-component "primitive equations" model introduced by Lorenz $[1,8]$.

Considering the matrix formulation of the Poisson bracket $\{\cdot, \cdot\}$, given in coordinates by

$$
\pi=\left[\begin{array}{ccccc}
0 & 0 & -x_{2} & 0 & b x_{2} \\
0 & 0 & x_{1} & 0 & -b x_{1} \\
x_{2} & -x_{1} & 0 & 0 & 0 \\
0 & 0 & 0 & 0 & -1 \\
-b x_{2} & b x_{1} & 0 & 1 & 0
\end{array}\right],
$$

system (1) has the Hamiltonian form [8]:

$$
\dot{x}=\{x, H\},
$$

where the Hamiltonian $H$ is given by (2). Hence $\left(\mathbb{R}^{5}, \pi, X_{H}\right)$ is a Hamilton-Poisson realization of dynamics (1), where

$$
\begin{aligned}
& X_{H}=\left(-x_{2} x_{3}+b x_{2} x_{5}, x_{1} x_{3}-b x_{1} x_{5},-x_{1} x_{2},-x_{5}, x_{4}\right. \\
& \left.\quad+b x_{1} x_{2}\right) .
\end{aligned}
$$

It is easy to see that the function $C$ is a Casimir for the above Poisson bracket.

In the following we study the stability of system (1).

The equilibrium states of system (1) are given as the union of the following families:

$$
\begin{aligned}
& \mathscr{E}_{1}=\{(M, 0,0,0,0) \mid M \in \mathbb{R}\}, \\
& \mathscr{E}_{2}=\left\{(0, M, 0,0,0) \mid M \in \mathbb{R}^{*}\right\}, \\
& \mathscr{E}_{3}=\left\{(0,0, M, 0,0) \mid M \in \mathbb{R}^{*}\right\} .
\end{aligned}
$$

Let $e_{M}^{1}=(M, 0,0,0,0) \in \mathscr{E}_{1}$. Considering the function $L \in$ $\mathscr{C}^{\infty}\left(\mathbb{R}^{5}, \mathbb{R}\right)$,

$$
\begin{aligned}
L\left(x_{1}, x_{2}, x_{3}, x_{4}, x_{5}\right)= & x_{2}^{2}+x_{3}^{2}+x_{4}^{2}+x_{5}^{2} \\
& +\left(x_{1}^{2}+x_{2}^{2}-M^{2}\right)^{2},
\end{aligned}
$$

we have

$$
\begin{aligned}
& L\left(e_{M}^{1}\right)=0, \\
& \begin{array}{l}
L\left(x_{1}, x_{2}, x_{3}, x_{4}, x_{5}\right)>0, \\
\quad(\forall)\left(x_{1}, x_{2}, x_{3}, x_{4}, x_{5}\right) \in V \backslash\left\{e_{M}^{1}\right\}, \\
\dot{L}\left(x_{1}, x_{2}, x_{3}, x_{4}, x_{5}\right) \\
\quad=2 x_{2} \dot{x}_{2}+2 x_{3} \dot{x}_{3}+2 x_{4} \dot{x}_{4}+2 x_{5} \dot{x}_{5} \\
\quad+4\left(x_{1}^{2}+x_{2}^{2}-M^{2}\right)\left(x_{1} \dot{x}_{1}+x_{2} \dot{x}_{2}\right)=0, \\
\quad(\forall)\left(x_{1}, x_{2}, x_{3}, x_{4}, x_{5}\right) \in V,
\end{array}
\end{aligned}
$$

for some neighbourhood $V$ of $e_{M}^{1}$.
By $[19,20]$, we deduce that all the equilibrium states from the family $\mathscr{E}_{1}$ are nonlinearly stable.

The characteristic polynomial associated with the linear part of system (1) at the equilibrium $e_{M}^{2}=(0, M, 0,0,0), M \neq$ 0 , is given by

$$
p_{e_{M}^{2}}(\lambda)=-\lambda\left[\lambda^{4}-\left(M^{2}+b^{2} M^{2}-1\right) \lambda^{2}-M^{2}\right] .
$$

We notice that a root of $p_{E_{M}^{2}}$ is strictly positive, whence $e_{M}^{2}$ is an unstable equilibrium state. Therefore, all the equilibrium states from the family $\mathscr{E}_{2}$ are unstable.

Let $e_{M}^{3} \in \mathscr{E}_{3}$. The roots of the characteristic polynomial associated with the linear part of system (1) at $e_{M}^{3}$ are

$$
\begin{aligned}
\lambda_{1} & =0, \\
\lambda_{2,3} & = \pm i, \\
\lambda_{4,5} & = \pm i M .
\end{aligned}
$$

Hence all the equilibrium states from the family $\mathscr{E}_{3}$ are spectrally stable.

Now, we study the existence of periodic orbits of system (1) around the equilibrium states from the family $\mathscr{E}_{1} \backslash$ $\{(0,0,0,0,0)\}$.

Since the eigenvalues of the linear part of system (1) at the equilibrium $e_{M}^{1}=(M, 0,0,0,0), M \neq 0$, are

$$
\begin{aligned}
\lambda_{1} & =0, \\
\lambda_{2,3} & = \pm i \sqrt{-y_{1}}, \\
\lambda_{4,5} & = \pm i \sqrt{-y_{2}},
\end{aligned}
$$

where $y_{1}$ and $y_{2}$ are the roots of the equation

$$
y^{2}+\left(M^{2}+b^{2} M^{2}+1\right) y+M^{2}=0,
$$

we apply Theorem 2.1 from [21]. The eigenspace corresponding to the eigenvalue $\lambda_{1}=0$ has one dimension. Taking the constant of motion $I: \mathbb{R}^{5} \rightarrow \mathbb{R}$,

$$
I\left(x_{1}, x_{2}, x_{3}, x_{4}, x_{5}\right)=x_{2}^{2}+x_{3}^{2}+x_{4}^{2}+x_{5}^{2},
$$

it follows that

$$
\begin{array}{r}
d I\left(e_{M}^{1}\right)=0, \\
\left.d^{2} I\left(e_{M}^{1}\right)\right|_{W \times W}>0,
\end{array}
$$

where

$$
\begin{gathered}
W=\operatorname{ker} d C\left(e_{M}^{1}\right)=\operatorname{Span}_{\mathbb{R}}\{(0,1,0,0,0),(0,0,1,0,0), \\
(0,0,0,1,0),(0,0,0,0,1)\} .
\end{gathered}
$$

Therefore, for each sufficiently small $\varepsilon \in \mathbb{R}_{+}^{*}$, any integral hypersurface

$$
\Sigma_{\varepsilon}^{e_{M}^{1}}: x_{2}^{2}+x_{3}^{2}+x_{4}^{2}+x_{5}^{2}=\varepsilon^{2}
$$




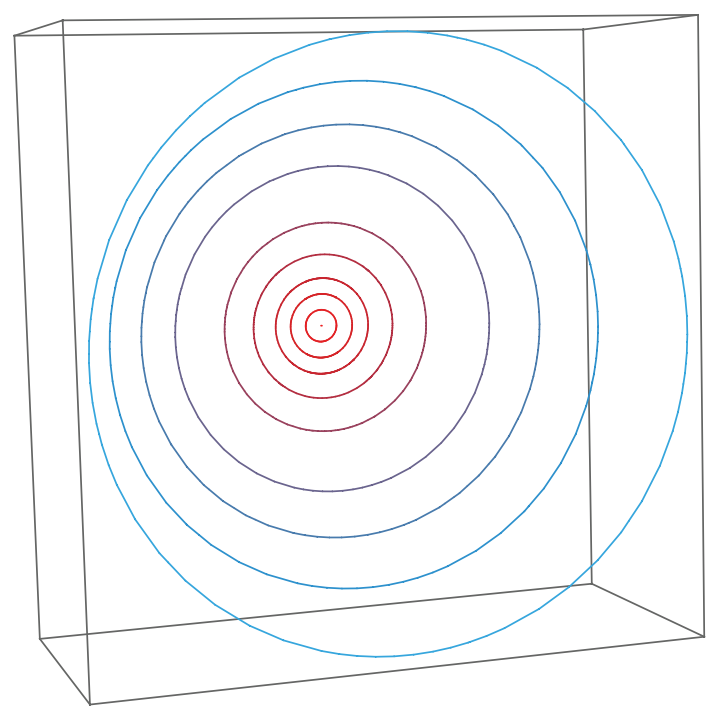

FIGURE 1: Periodic orbits around equilibrium state $(0,0, M, 0,0)(3 \mathrm{D}$ view in the $x_{3}-x_{4}-x_{5}$ space).

contains at least one periodic orbit of system (1) whose period is close to $2 \pi / \sqrt{-y_{1}}$ and at least one periodic orbit of system (1) whose period is close to $2 \pi / \sqrt{-y_{2}}$.

In the case of the equilibrium states from $\mathscr{E}_{3}$, we cannot apply the above method. On the other hand the dynamics of system (1) are carried out at the intersection of the hypersurfaces

$$
\begin{aligned}
& H\left(x_{1}, x_{2}, x_{3}, x_{4}, x_{5}\right)=H(0,0, M, 0,0), \\
& C\left(x_{1}, x_{2}, x_{3}, x_{4}, x_{5}\right)=C(0,0, M, 0,0) ;
\end{aligned}
$$

that is,

$$
\begin{aligned}
x_{3}^{2}+x_{4}^{2}+x_{5}^{2} & =M^{2}, \\
x_{1}^{2}+x_{2}^{2} & =0 .
\end{aligned}
$$

Then the solution of system (1) is

$$
\begin{aligned}
& x_{1}=0, \\
& x_{2}=0, \\
& x_{3}= \pm \sqrt{M^{2}-k_{1}^{2}-k_{2}^{2}}, \\
& x_{4}=k_{1} \cos t+k_{2} \sin t, \\
& x_{5}=k_{1} \sin t-k_{2} \cos t,
\end{aligned}
$$

where $k_{1}, k_{2} \in \mathbb{R}, k_{1}^{2}+k_{2}^{2} \leq M^{2}$. We remark that (20) represents periodic orbits around equilibrium state $(0,0, M, 0,0), M \in \mathbb{R}^{*}$ (see Figure 1 ).

\section{Symplectic Realization and Symmetries}

First result shows that system (1) can be regarded as a Hamiltonian mechanical system.
Theorem 1. The Hamilton-Poisson mechanical system $\left(\mathbb{R}^{5}, \pi, X_{H}\right)$ has a full symplectic realization $\left(T^{*} \mathbb{R}^{3} \simeq\right.$ $\left.\mathbb{R}^{6}, \omega, X_{\widetilde{H}}\right)$, where

$$
\begin{aligned}
& \omega=d p_{1} \wedge d q_{1}+d p_{2} \wedge d q_{2}+d p_{3} \wedge d q_{3}, \\
& \widetilde{H}=\frac{1}{2}\left(p_{1}^{2}+p_{2}^{2}+p_{3}^{2}\right)+\frac{1}{2}\left(q_{2}-b p_{1}\right)^{2}+\frac{1}{2} p_{3}^{2} \sin ^{2} q_{1},
\end{aligned}
$$

and the corresponding Hamiltonian vector field is as follows:

$$
\begin{aligned}
X_{\widetilde{H}}= & {\left[\left(1+b^{2}\right) p_{1}-b q_{2}\right] \frac{\partial}{\partial q_{1}}+p_{2} \frac{\partial}{\partial q_{2}} } \\
& +\left(p_{3}+p_{3} \sin ^{2} q_{1}\right) \frac{\partial}{\partial q_{3}}-p_{3}^{2} \sin q_{1} \cos q_{1} \frac{\partial}{\partial p_{1}} \\
& +\left(b p_{1}-q_{2}\right) \frac{\partial}{\partial p_{2}} .
\end{aligned}
$$

Proof. Using the Hamiltonian $\widetilde{H}$ one obtains the corresponding Hamilton's equations:

$$
\begin{aligned}
& \dot{q}_{1}=\left(1+b^{2}\right) p_{1}-b q_{2}, \\
& \dot{q}_{2}=p_{2}, \\
& \dot{q}_{3}=p_{3}+p_{3} \sin ^{2} q_{1}, \\
& \dot{p}_{1}=-p_{3}^{2} \sin q_{1} \cos q_{1}, \\
& \dot{p}_{2}=b p_{1}-q_{2}, \\
& \dot{p}_{3}=0 .
\end{aligned}
$$

We consider the mapping $\varphi: \mathbb{R}^{6} \rightarrow \mathbb{R}^{5}$,

$$
\begin{aligned}
\varphi & \left(q_{1}, q_{2}, q_{3}, p_{1}, p_{2}, p_{3}\right) \\
& =\left(p_{3} \cos q_{1}, p_{3} \sin q_{1}, p_{1}, p_{2}, q_{2}-b p_{1}\right) \\
& =\left(x_{1}, x_{2}, x_{3}, x_{4}, x_{5}\right) .
\end{aligned}
$$

Using the standard symplectic bracket

$$
\{f, g\}_{\omega}=\sum_{i=1}^{3}\left(\frac{\partial f}{\partial q_{i}} \cdot \frac{\partial g}{\partial p_{i}}-\frac{\partial f}{\partial p_{i}} \cdot \frac{\partial g}{\partial q_{i}}\right)
$$

one gets

$$
\begin{aligned}
& \left\{x_{1}, x_{2}\right\}_{\omega}=0, \\
& \left\{x_{1}, x_{3}\right\}_{\omega}=-x_{2},
\end{aligned}
$$




$$
\begin{aligned}
& \left\{x_{1}, x_{4}\right\}_{\omega}=0, \\
& \left\{x_{1}, x_{5}\right\}_{\omega}=b x_{2}, \\
& \left\{x_{2}, x_{3}\right\}_{\omega}=x_{1}, \\
& \left\{x_{2}, x_{4}\right\}_{\omega}=0, \\
& \left\{x_{2}, x_{5}\right\}_{\omega}=-b x_{1}, \\
& \left\{x_{3}, x_{4}\right\}_{\omega}=0, \\
& \left\{x_{3}, x_{5}\right\}_{\omega}=0, \\
& \left\{x_{4}, x_{5}\right\}_{\omega}=-1 .
\end{aligned}
$$

Hence the canonical structure $\{\cdot, \cdot\}_{\omega}$ is mapped onto the Poisson structure $\pi$.

Taking into account relations (23), we have

$$
\begin{aligned}
& \dot{x}_{1}=-p_{3} \sin q_{1} \cdot \dot{q}_{1}=-x_{2} x_{3}+b x_{2} x_{5}, \\
& \dot{x}_{2}=p_{3} \cos q_{1} \cdot \dot{q}_{1}=x_{1} x_{3}-b x_{1} x_{5}, \\
& \dot{x}_{3}=\dot{p}_{1}=-x_{1} x_{2}, \\
& \dot{x}_{4}=\dot{p}_{2}=-x_{5}, \\
& \dot{x}_{5}=\dot{q}_{2}-b \dot{p}_{1}=x_{4}+b x_{1} x_{2} .
\end{aligned}
$$

Therefore the Hamiltonian vector field $X_{\widetilde{H}}$ is mapped onto the Hamiltonian vector field $X_{H}$. Moreover $\varphi$ is a surjective submersion and $H \circ \varphi=\widetilde{H}$, which finishes the proof.

Denoting $\widetilde{C}:=C \circ \varphi$, it follows that $\widetilde{C}=p_{3}$.

The next result states that system (23) can be written in Lagrangian formalism.

Theorem 2. System (23) has the form

$$
\begin{array}{r}
\ddot{q}_{1}+\left(1+b^{2}\right) \frac{\sin q_{1} \cos q_{1}}{\left(1+\sin ^{2} q_{1}\right)^{2}} \dot{q}_{3}^{2}+b \dot{q}_{2}=0, \\
\ddot{q}_{2}-\frac{b}{1+b^{2}} \dot{q}_{1}+\frac{1}{1+b^{2}} q_{2}=0, \\
\ddot{q}_{3}-\frac{2 \sin q_{1} \cos q_{1}}{1+\sin ^{2} q_{1}} \dot{q}_{1} \dot{q}_{3}=0,
\end{array}
$$

on the tangent bundle $T \mathbb{R}^{3}$. Also, system (28) represents the Euler-Lagrange equations generated by the Lagrangian

$$
\begin{aligned}
L= & \frac{1}{2\left(1+b^{2}\right)} \dot{q}_{1}^{2}+\frac{1}{2} \dot{q}_{2}^{2}+\frac{1}{2\left(1+\sin ^{2} q_{1}\right)} \dot{q}_{3}^{2} \\
& +\frac{b}{1+b^{2}} \dot{q}_{1} q_{2}-\frac{1}{2\left(1+b^{2}\right)} q_{2}^{2} .
\end{aligned}
$$

Proof. By Hamilton's equations (23) we obtain

$$
\begin{aligned}
& p_{1}=\frac{1}{1+b^{2}} \dot{q}_{1}+\frac{b}{1+b^{2}} q_{2} \\
& p_{2}=\dot{q}_{2} \\
& p_{3}=\frac{1}{1+\sin ^{2} q_{1}} \dot{q}_{3},
\end{aligned}
$$

whence

$$
\begin{aligned}
& \dot{p}_{1}=\frac{1}{1+b^{2}} \ddot{q}_{1}+\frac{b}{1+b^{2}} \dot{q}_{2}, \\
& \dot{p}_{2}=\ddot{q}_{2}, \\
& \dot{p}_{3}=\frac{-2 \sin q_{1} \cos q_{1}}{\left(1+\sin ^{2} q_{1}\right)^{2}} \dot{q}_{1} \dot{q}_{3}+\frac{1}{1+\sin ^{2} q_{1}} \ddot{q}_{3} .
\end{aligned}
$$

Substituting $p_{1}, p_{2}, p_{3}, \dot{p}_{1}, \dot{p}_{2}, \dot{p}_{3}$ into (23), one gets (28). For the Lagrangian $L$ given by (29), the Euler-Lagrange equations,

$$
\frac{d}{d t}\left(\frac{\partial L}{\partial \dot{q}_{i}}\right)-\frac{\partial L}{\partial q_{i}}=0, \quad i \in\{1,2,3\},
$$

have the form (28). The relation between the Hamiltonian $\widetilde{H}$ and the Lagrangian $L$,

$$
\widetilde{H}=\sum_{i=1}^{3} p_{i} \dot{q}_{i}-L,
$$

where

$$
p_{i}=\frac{\partial L}{\partial \dot{q}_{i}}, \quad i \in\{1,2,3\},
$$

follows by relations (23).

For details about Lagrangian and Hamiltonian formalism see, for example, $[22,23]$.

In the sequel we study the Lie-point symmetries for EulerLagrange equations (28).

We recall that a vector field

$$
\begin{aligned}
\mathbf{v}= & \xi\left(q_{1}, q_{2}, q_{3}, t\right) \frac{\partial}{\partial t}+\eta_{1}\left(q_{1}, q_{2}, q_{3}, t\right) \frac{\partial}{\partial q_{1}} \\
& +\eta_{2}\left(q_{1}, q_{2}, q_{3}, t\right) \frac{\partial}{\partial q_{2}}+\eta_{3}\left(q_{1}, q_{2}, q_{3}, t\right) \frac{\partial}{\partial q_{3}}
\end{aligned}
$$

is a Lie-point symmetry for Euler-Lagrange equations if the action of its second prolongation on these equations vanishes. For more details about symmetries see, for example, [24-26]. Applying the second prolongation of $\mathbf{v}$,

$$
\begin{aligned}
\operatorname{pr}^{(2)}(\mathbf{v})= & \mathbf{v}+\sum_{i=1}^{3}\left(\dot{\eta}_{i}-\dot{\xi} \dot{q}_{i}\right) \frac{\partial}{\partial \dot{q}_{i}} \\
& +\sum_{i=1}^{3}\left(\ddot{\eta}_{i}-\ddot{\xi}_{\dot{q}_{i}}-2 \dot{\xi} \ddot{q}_{i}\right) \frac{\partial}{\partial \ddot{q}_{i}},
\end{aligned}
$$


on (28) one obtains

$$
\begin{aligned}
& \ddot{\eta}_{1}-\ddot{\xi}_{\dot{q}_{1}}-2 \ddot{q}_{1} \dot{\xi} \\
& +\left(1+b^{2}\right) \frac{1-5 \sin ^{2} q_{1}+2 \sin ^{4} q_{1}}{\left(1+\sin ^{2} q_{1}\right)^{3}} \dot{q}_{3}^{2} \eta_{1} \\
& +b\left(\dot{\eta}_{2}-\dot{\xi} \dot{q}_{2}\right) \\
& +2\left(1+b^{2}\right) \frac{\sin q_{1} \cos q_{1}}{\left(1+\sin ^{2} q_{1}\right)^{2}}\left(\dot{\eta}_{3}-\dot{\xi} \dot{q}_{3}\right) \dot{q}_{3}=0, \\
& \ddot{\eta}_{2}-\ddot{\xi}_{\dot{q}_{2}}-2 \ddot{q}_{2} \dot{\xi}+\frac{1}{1+b^{2}} \eta_{2}-\frac{b}{1+b^{2}}\left(\dot{\eta}_{1}-\dot{\xi}_{\dot{q}_{1}}\right)=0 \text {, } \\
& \ddot{\eta}_{3}-\ddot{\xi} \dot{q}_{3}-2 \ddot{q}_{3} \dot{\xi}+2 \frac{3 \sin ^{2} q_{1}-1}{\left(1+\sin ^{2} q_{1}\right)^{2}} \eta_{1} \dot{q}_{1} \dot{q}_{3} \\
& -\frac{2 \sin q_{1} \cos q_{1}}{1+\sin ^{2} q_{1}}\left(\dot{\eta}_{1}-\dot{\xi} \dot{q}_{1}\right) \dot{q}_{3} \\
& -\frac{2 \sin q_{1} \cos q_{1}}{1+\sin ^{2} q_{1}}\left(\dot{\eta}_{3}-\dot{\xi} \dot{q}_{3}\right) \dot{q}_{1}=0 .
\end{aligned}
$$

The resulting equations obtained by expanding $\dot{\xi}, \ddot{\xi}, \dot{\eta}_{1}, \ddot{\eta}_{1}$, $\dot{\eta}_{2}, \ddot{\eta}_{2}, \dot{\eta}_{3}, \ddot{\eta}_{3}$ and replacing $\ddot{q}_{1}, \ddot{q}_{2}$, and $\ddot{q}_{3}$ must be satisfied identically in $t, q_{1}, q_{2}, q_{3}, \dot{q}_{1}, \dot{q}_{2}, \dot{q}_{3}$, which are all independent variables.

In the case $b \neq 0$, it follows that

$$
\begin{aligned}
& \xi_{q_{1}}=\xi_{q_{2}}=\xi_{q_{3}}=0, \\
& \eta_{1, t}=\eta_{1, q_{2}}=\eta_{1, q_{3}}=0, \\
& \eta_{1, q_{1} q_{1}}=0, \\
& \eta_{2, q_{1}}=\eta_{2, q_{3}}=0, \\
& \eta_{2, q_{2} q_{2}}=0, \\
& \eta_{3, t}=\eta_{3, q_{1}}=\eta_{3, q_{2}}=0, \\
& \eta_{3, q_{3} q_{3}}=0, \\
& \eta_{2}+\left(1+b^{2}\right) \eta_{2, t t}-q_{2} \eta_{2, q_{2}}+2 q_{2} \xi_{t}=0, \\
& \eta_{1, q_{1}}+\xi_{t}-\eta_{2, q_{2}}=0, \\
& 2 \eta_{2, t q_{2}}-\xi_{t t}=0, \\
& b \eta_{2, t}=0, \\
& b\left(\eta_{2, q_{2}}+\xi_{t}-\eta_{1, q_{1}}\right)=0, \\
& \frac{1-5 \sin ^{2} q_{1}+2 \sin ^{4} q_{1} \eta_{1}}{1+\sin ^{2} q_{1}} \\
& +\sin _{1} q_{1} \cos _{1} q_{1}-2 \\
& 1+\sin ^{2} q_{1} \\
& \left.\frac{6}{1} \eta_{3, q_{3}}-\eta_{1, q_{1}}\right)=0, \\
& \sin q_{1} \cos q_{1} \eta_{1, q_{1}}=0 .
\end{aligned}
$$

The last relation implies $\eta_{1}=0$. It results in

$$
\begin{aligned}
\xi & =\alpha, \\
\eta_{1} & =0, \\
\eta_{2} & =0, \\
\eta_{3} & =\beta,
\end{aligned}
$$

$\alpha, \beta \in \mathbb{R}$.

In the case $b=0$, it follows that

$$
\begin{aligned}
\xi_{q_{1}} & =\xi_{q_{2}}=\xi_{q_{3}}=0, \\
\eta_{1} & =0, \\
\eta_{2, q_{1}} & =\eta_{2, q_{3}}=0, \\
\eta_{2, q_{2} q_{2}} & =0, \\
\eta_{2, t q_{2}} & =0, \\
\eta_{3, t} & =\eta_{3, q_{1}}=\eta_{3, q_{2}}=\eta_{3, q_{3}}=0, \\
\eta_{2}+\eta_{2, t t}-q_{2} \eta_{2, q_{2}}+2 q_{2} \xi_{t} & =0 .
\end{aligned}
$$

It results in

$$
\begin{aligned}
\xi & =a t+b, \\
\eta_{2} & =c q_{2}+f(t),
\end{aligned}
$$

$a, b, c \in \mathbb{R}$.

Then $a=0$ and $f^{\prime \prime}(t)+f(t)=0$. Therefore

$$
\begin{aligned}
\xi & =\alpha, \\
\eta_{1} & =0, \\
\eta_{2} & =\gamma q_{2}+\delta \cos t+\theta \sin t, \\
\eta_{3} & =\beta,
\end{aligned}
$$

$\alpha, \beta, \gamma, \delta, \theta \in \mathbb{R}$.

We can conclude the following result.

Theorem 3. The symmetries of (28) are given by

$$
\mathbf{v}=\alpha \frac{\partial}{\partial t}+\beta \frac{\partial}{\partial q_{3}},
$$

where $\alpha, \beta \in \mathbb{R}$, in the case $b \in \mathbb{R}^{*}$, respectively, and

$$
\mathbf{u}=\alpha \frac{\partial}{\partial t}+\left(\gamma q_{2}+\delta \cos t+\theta \sin t\right) \frac{\partial}{\partial q_{2}}+\beta \frac{\partial}{\partial q_{3}},
$$

where $\alpha, \beta, \gamma, \delta, \theta \in \mathbb{R}$, in the case $b=0$.

Remark 4. Let $\gamma=\delta=\theta=0$. Denoting $\mathbf{v}_{1}=\partial / \partial t$ and $\mathbf{v}_{2}=\partial / \partial q_{3}$, it follows that $\mathbf{v}_{1}, \mathbf{v}_{2}$ are variational symmetries. Moreover,

(i) for $\beta=0$ and $\alpha \neq 0$, we have $\mathbf{v}=\alpha \mathbf{v}_{1}$ that represents the time translation symmetry which generates the conservation of energy $\widetilde{H}$; 
(ii) for $\alpha=0$ and $\beta \neq 0$, we have $\mathbf{v}=\beta \mathbf{v}_{2}$ that represents a translation in the cyclic $q_{3}$ direction which is related to the conservation of $p_{3}$.

We notice that the vector field $\mathbf{u}$ leads to the vector field

$$
\begin{aligned}
\mathbf{X}= & \alpha \frac{\partial}{\partial t}+\left(\gamma x_{4}-\delta \sin t+\theta \cos t\right) \frac{\partial}{\partial x_{4}} \\
& +\left(\gamma x_{5}+\delta \cos t+\theta \sin t\right) \frac{\partial}{\partial x_{5}} .
\end{aligned}
$$

Also, we can consider the vector field

$$
\begin{aligned}
\mathbf{Y}= & \alpha \frac{\partial}{\partial t}+\left(\gamma x_{5}-\delta \sin t+\theta \cos t\right) \frac{\partial}{\partial x_{4}} \\
& +\left(-\gamma x_{4}+\delta \cos t+\theta \sin t\right) \frac{\partial}{\partial x_{5}} .
\end{aligned}
$$

The last result furnishes some symmetries of system (1) in the case $b=0$.

Proposition 5. The vector field $\mathbf{X}$ given by (45) is a Lie-point symmetry of system (1) in the case $b=0$. Also, if $\delta=\theta=0$, then $\mathbf{X}$ is a symmetry of system (1) in the case $b=0$. Moreover, the vector field $\mathbf{Y}$ given by (46) has the same properties.

Proof. It is easy to see that the action of the first prolongation of $\mathbf{X}$ on (1) in the case $b=0$ vanishes. Therefore $\mathbf{X}$ is a Liepoint symmetry.

Considering $\delta=\theta=0$, it immediately follows that

$$
\frac{\partial \mathbf{X}}{\partial t}+\left[\mathbf{X}, \mathbf{X}_{f}\right]=0
$$

where

$$
\mathbf{X}_{f}=\left(-x_{2} x_{3}, x_{1} x_{3},-x_{1} x_{3},-x_{5}, x_{4}\right),
$$

whence $\mathbf{X}$ is a symmetry of system (1) in the case $b=0$.

\section{Conclusions}

In this paper the 5-dimensional Lorenz system is considered. This is a system of five differential equations which couples the Rossby waves and gravity waves. In Section 2 some stability problems and the existence of periodic orbits are studied. The equilibrium states of considered system are given as the union of three families of points. For one of these families, all the equilibria are spectrally stable, but it remains an open problem to establish if these equilibria are nonlinearly stable. In the third part of the paper a symplectic realization and the corresponding Lagrangian formulation are given. In the last part of our work, some symmetries of the 5-dimensional Lorenz system are studied. Knowing the connection between symmetries and conservative laws, we tried to determine a third first integral of the considered system, provided that it exists.

\section{Conflict of Interests}

The authors declare that there is no conflict of interests regarding the publication of this paper.

\section{References}

[1] E. N. Lorenz, "On the existence of a slow manifold," Journal of the Atmospheric Sciences, vol. 43, no. 15, pp. 1547-1558, 1986.

[2] E. N. Lorenz and V. Krishnamurthy, "On the nonexistence of a slow manifold," Journal of the Atmospheric Sciences, vol. 44, no. 20, pp. 2940-2950, 1987.

[3] J. P. Boyd, “The slow manifold of a five-mode model," Journal of the Atmospheric Sciences, vol. 51, no. 8, pp. 1057-1064, 1994.

[4] R. Camassa and S.-K. Tin, "The global geometry of the slow manifold in the Lorenz-Krishnamurthy model," Journal of the Atmospheric Sciences, vol. 53, no. 22, pp. 3251-3264, 1996.

[5] J.-M. Ginoux, "The slow invariant manifold of the LorenzKrishnamurty model," Qualitative Theory of Dynamical Systems, vol. 13, no. 1, pp. 19-37, 2014.

[6] O. Bokhove, "On Hamiltonian balanced models," in Proceedings of the 9th Conference on Atmospheric and Oceanic Waves and Stability, pp. 367-368, American Meteorological Society, San Antonio, Tex, USA, 1993.

[7] R. Camassa, "On the geometry of an atmospheric slow manifold," Physica D: Nonlinear Phenomena, vol. 84, no. 3-4, pp. 357397, 1995.

[8] O. Bokhove and T. G. Shepherd, "On Hamiltonian balanced dynamics and the slowest invariant manifold," Journal of the Atmospheric Sciences, vol. 53, no. 2, pp. 276-297, 1996.

[9] P. Birtea, M. Puta, T. S. Ratiu, and R. Tudoran, "A short proof of chaos in an atmospheric system," Physics Letters A, vol. 300, no. 2-3, pp. 189-191, 2002.

[10] J. Llibre, R. Saghin, and X. Zhang, "On the analytic integrability of the 5-dimensional lorenz system for the gravity-wave activity," Proceedings of the American Mathematical Society, vol. 142, no. 2, pp. 531-537, 2014.

[11] E. Noether, "Invariante variations probleme," Nachrichten von der Gesellschaft der Wissenschaften zu Göttingen, MathematischPhysikalische Klasse, vol. 1, pp. 235-257, 1918, English translation: Transport Theory and Statistical Physics, vol. 1, pp. 186-207, 1971.

[12] I. Caşu, "Symmetries of the Maxwell-Bloch equations with the rotating wave approximation," Regular and Chaotic Dynamics, vol. 19, no. 5, pp. 548-555, 2014.

[13] A. Bihlo, Symmetry methods in the atmospheric sciences [Ph.D. thesis], Universitat Wien, 2010.

[14] M. L. Gandarias, M. S. Bruzón, and M. Rosa, "Symmetries and conservation laws for some compacton equation," Mathematical Problems in Engineering, vol. 2015, Article ID 430823, 6 pages, 2015.

[15] R. J. Gray, "The Lie point symmetry generators admitted by systems of linear differential equations," Proceedings of the Royal Society A: Mathematical, Physical and Engineering Sciences, vol. 470, no. 2166, 12 pages, 2014.

[16] R. Naz, I. Naeem, and F. M. Mahomed, "A partial lagrangian approach to mathematical models of epidemiology," Mathematical Problems in Engineering, vol. 2015, Article ID 602915, 11 pages, 2015.

[17] Z. Cao and Y. Lin, "Lie point symmetries, conservation laws, and solutions of a space dependent reaction-diffusion equation," Applied Mathematics and Computation, vol. 248, no. 1, pp. 386-398, 2014.

[18] P. Holmes and E. T. Shea-Brown, "Stability," Scholarpedia, vol. 1, no. 10 , article $1838,2006$. 
[19] A. M. Lyapunov, "Problème général de la stabilité du mouvement," Annales de la Faculté des Sciences de Toulouse, vol. 9, pp. 203-474, 1907, Kharkov 1892.

[20] A. M. Lyapunov, Problème Général de la Stabilité du Mouvement, vol. 17, Princeton University Press, Princeton, NJ, USA, 1949.

[21] P. Birtea, M. Puta, and R. M. Tudoran, "Periodic orbits in the case of a zero eigenvalue," Comptes Rendus Mathematique, vol. 344, no. 12, pp. 779-784, 2007.

[22] P. Libermann and C.-M. Marle, Symplectic Geometry and Analytical Mechanics, D. Reidel Publishing, Dordrecht, The Netherlands, 1987.

[23] J. E. Marsden and T. S. Raţiu, Introduction to Mechanics and Symmetry, vol. 17 of Texts in Applied Mathematics, Springer, Berlin, Germany, 2nd edition, 1999.

[24] G. W. Bluman and S. Kumei, Symmetries and Differential Equations, vol. 81 of Applied Mathematical Sciences, Springer, New York, NY, USA, 1989.

[25] P. J. Olver, Applications of Lie Groups to Differential Equations, Springer, New York, NY, USA, 1986.

[26] C. Lăzureanu and T. Bînzar, "Symmetries of some classes of dynamical systems," Journal of Nonlinear Mathematical Physics, vol. 22, no. 2, pp. 265-274, 2015. 


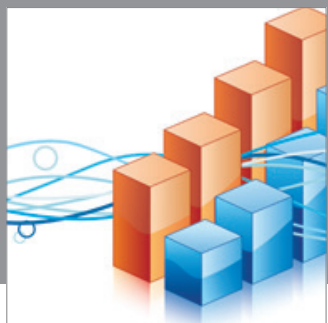

Advances in

Operations Research

mansans

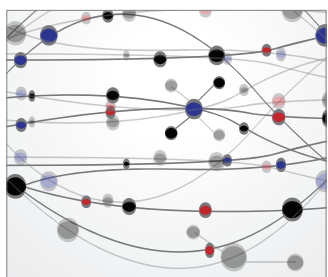

The Scientific World Journal
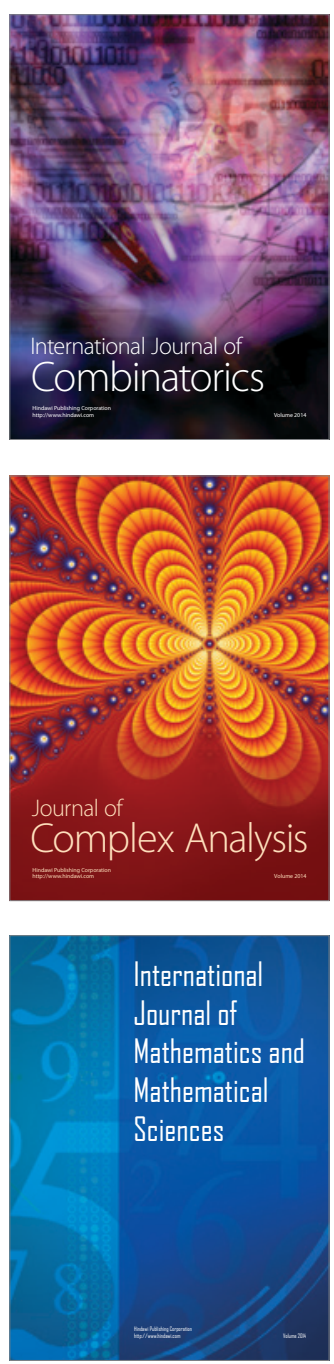
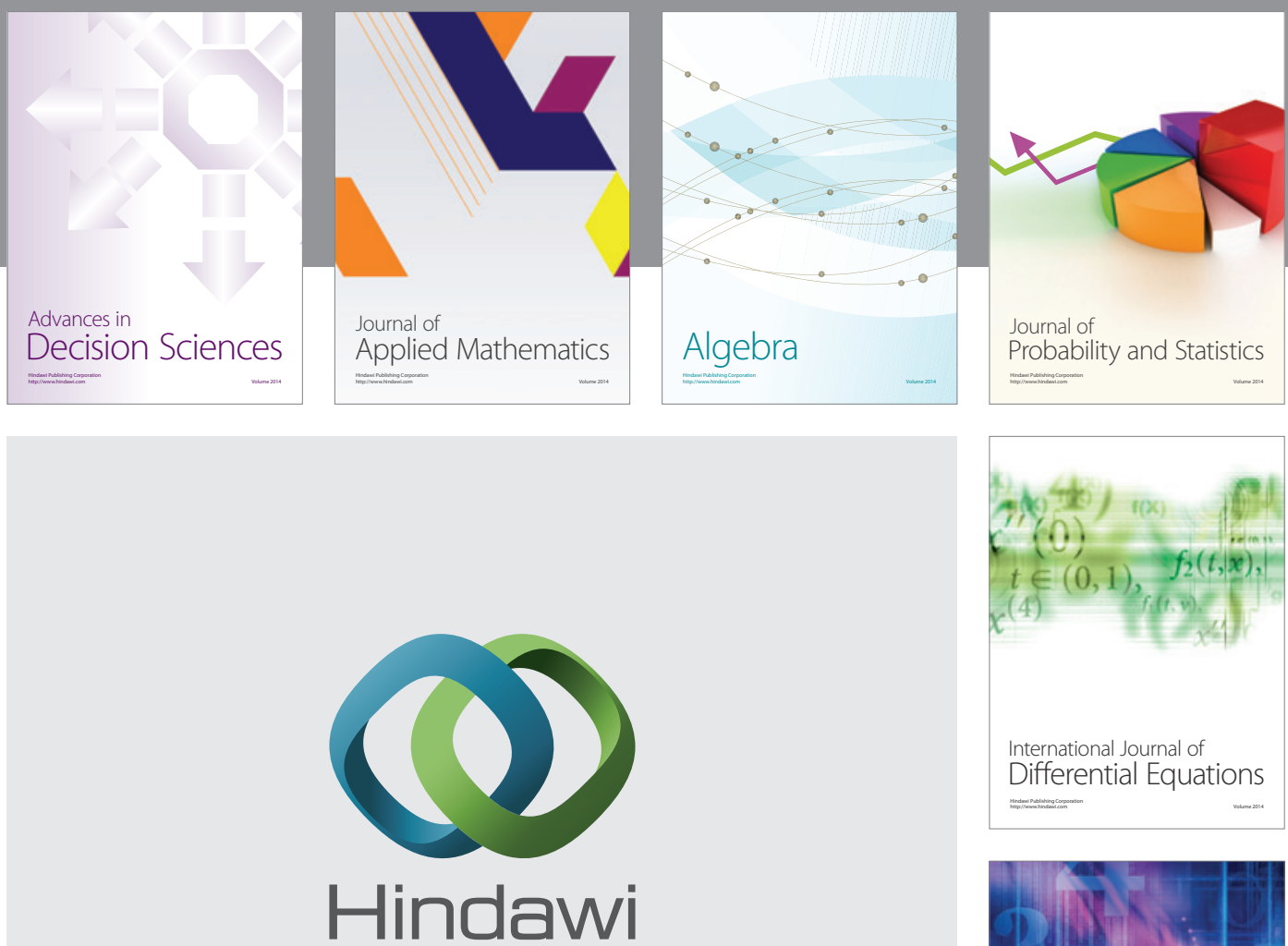

Submit your manuscripts at http://www.hindawi.com
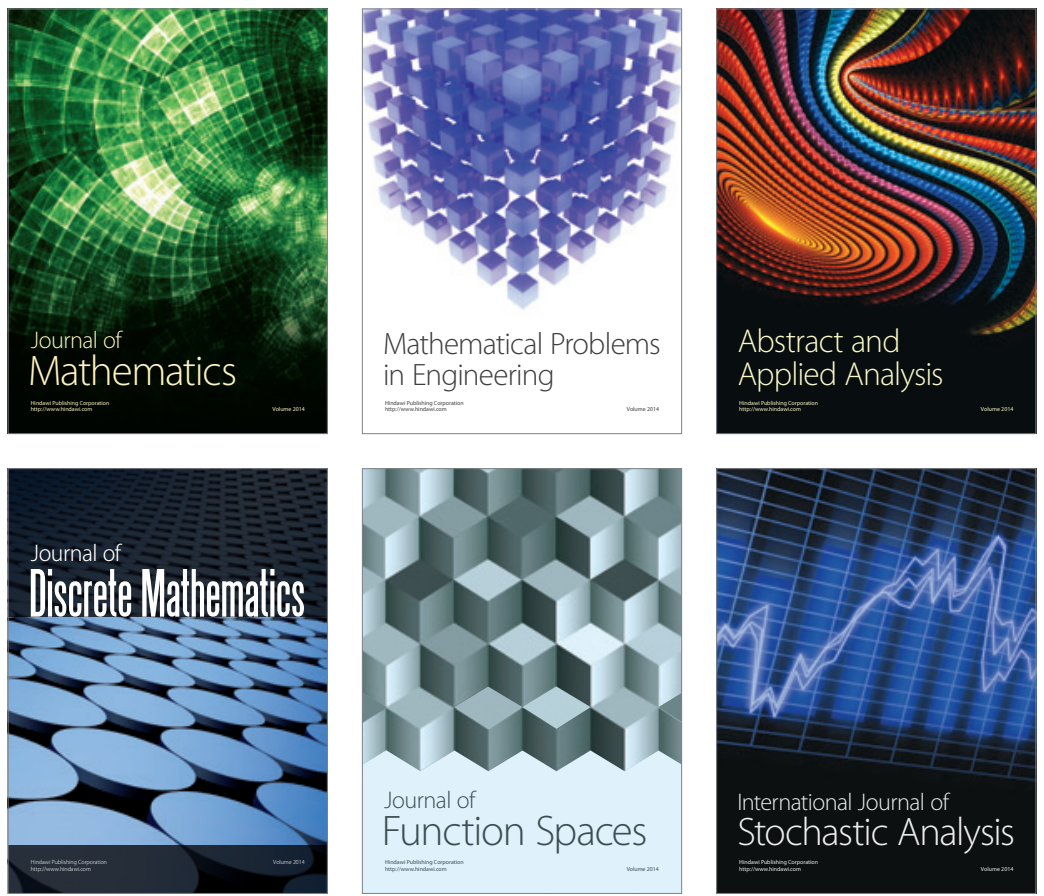

Journal of

Function Spaces

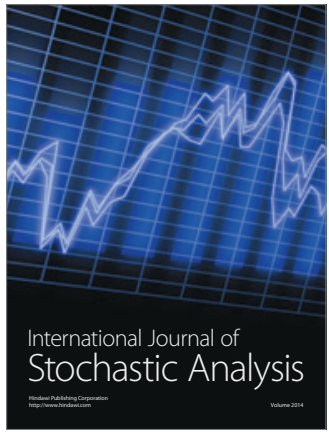

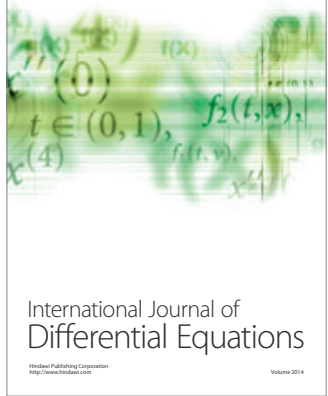
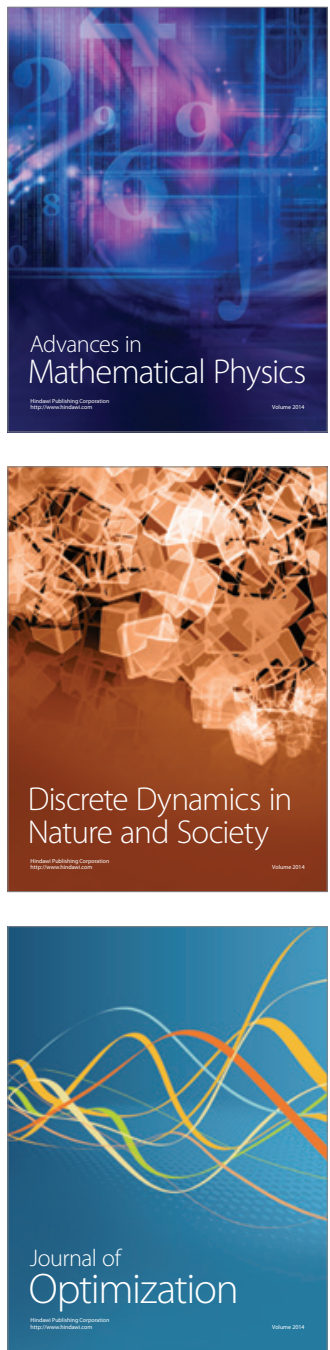